\title{
Peso, estatura e índice de masa corporal de niños y adolescentes de moderada altitud de Colombia
}

\author{
Weight, height and body mass index of children and adolescents \\ living at moderate altitude in Colombia
}

Mg. Edilberto Díaz Bonilla ${ }^{a}, M g$. Claudia L. Torres Galvisa, Dra. Rossana Gómez Campos b,c, Dr. Miguel de Arrudac , Dr. Jaime Pacheco Carrillo ${ }^{d}$ y Dr. Marco Cossio Bolaños ${ }^{c, e f}$

\section{RESUMEN}

Introducción. Existe preocupación progresiva por estudiar el crecimiento físico de diversas regiones del mundo, aunque no se considera la altitud como factor de ajuste.

Objetivos. Comparar variables de crecimiento físico y patrones deíndice de masa corporal (IMC) con la referencia del Centro para el Control y Prevención de Enfermedades 2012 (CDC según siglas en inglés) y desarrollar percentiles para niños y adolescentes .

Metodología. Se investigaron escolares de moderada altitud de Bogotá (Colombia). Se evaluó el peso, la estatura y se calculó el IMC. Las variables antropométricas fueron comparadas con referencia al CDC-2012, Brasil, Perú y Argentina.Se construyeron curvas por el método LMS (least-mean-square algorithm).

Resultados. Se estudió a 2241 escolares (1159 mujeres), entre 6,0 y 17,9 años. No hubo diferencias significativas en el peso e IMC entre 6 y 8 años con relación al CDC-2012; sin embargo, desde los 9 a 17 años, esta muestra evidenció valores inferiores de peso e IMC en relación con el CDC-2012. En la estatura, en ambos sexos, se mostraron valores inferiores del CDC-2012. Las comparaciones con las curvas regionales de Argentina, Perú y Brasil fueron relativamente similares, excepto en el IMC en mujeres, que presentron valores inferiores desde 13 a 17 años. Conclusión. Las variables de crecimiento de los escolares fueron inferiores con referencia al CDC-2012. Hubo ligeras discrepancias en el crecimiento físico y en el IMC con las curvas de Argentina, Brasily Perú. Se construyeron curvas para evaluar el crecimiento y el IMC de escolares de moderada altitud de Colombia.

Palabras clave: Crecimiento y desarrollo, niño, adolescente, altitud.

http:/ / dx.doi.org/10.5546/ aap.2018.e241

Texto completo en inglés:

http: / / dx.doi.org/10.5546/ aap.2018.eng.e241

Financiamiento:

Ninguno.

Conflicto de intereses:

Ninguno que declarar.

Recibido: 4-5-2017

Aceptado: 19-9-2017
Cómo citar: Díaz Bonilla E, Torres Galvis CL, Gómez Campos R, et al. Peso, estatura e índice de masa corporal de niños y adolescentes de moderada altitud de Colombia. Arch Argent Pediatr 2018;116(2):e241-e250.

\section{INTRODUCCIÓN}

El crecimiento físico (CF) y el desarrollo humano se caracterizan por su heterogeneidad intrapoblacional e interpoblacional. ${ }^{1}$ Se produce debido a la interacción continua y compleja de los genes entre sí y con diferentes condiciones mesológicas. ${ }^{2}$

De hecho, estudiar el CF entre poblaciones es relevante, pues ha sido, durante mucho tiempo, el objetivo primario en los ámbitos de la biología humana y la salud pública, ${ }^{0}$ inclusive, se ha sugerido que el peso y la estatura son indicadores clásicos importantes para evaluar el CF de niños y adolescentes. ${ }^{3}$

En ese contexto, son pocos los estudios efectuados en Colombia ${ }^{4}$ que han dado prioridad al estudio del CF en diversas regiones geográficas, sin considerar la altitud como variable de ajuste. De hecho, la altitud es una variable importante que juega un papel relevante en el estudio del CF, la cual ha sido ampliamente estudiada en diversas investigaciones, 5,6 inclusive, algunos estudios efectuados a moderada altitud han evidenciado diferencias significativas en los patrones del CF de niños y adolescentes. ${ }^{7}$

Esta información sugiere la influencia de las diferencias ambientales, culturales y genéticas específicas para cada población, por lo que es probable que los niños y adolescentes de moderada altitud de Bogotá (Colombia) puedan diferir en peso, estatura e IMC en relación con 
la referencia americana de los Centros para el Control y la Prevención de Enfermedades 2012 (CDC-2012) y otras referencias regionales de América del Sur.

En general, los estudios regionales que tienen como objetivo comparar el CF con referencias internacionales utilizan las propuestas de los $\mathrm{CDC}^{8,9}$ y la Organización Mundial de la Salud (OMS). ${ }^{10}$ Estas referencias, en esencia, son herramientas valiosas para la práctica clínica, para la evaluación individual y para el seguimiento del $\mathrm{CF}$. Inclusive, existe una preocupación adicional en relación con la salud pública de la obesidad infantil, ${ }^{11}$ puesto que, en los últimos años, se ha ido incrementando en niños y adolescentes en América del Sur, debido al fenómeno de la transición nutricional. ${ }^{12}$

Por lo tanto, este estudio tiene los siguientes objetivos: a) comparar las variables de crecimiento (peso y estatura) y los patrones de IMC con la referencia de los CDC-2012 y otros estudios internacionales; $\mathrm{y} \mathrm{b}$ ) desarrollar percentiles para el peso, estatura e IMC para niños y adolescentes escolares que viven a moderada altitud de Colombia.

\section{METODOLOGÍA}

\section{Tipo de estudio y muestra}

Se efectuó un estudio descriptivo transversal en escolares que vivían a moderada altitud en Bogotá (Colombia). El rango de edad osciló entre 6,0 y 17,9 años. Los escolares fueron reclutados de una institución educativa pública localizada a 2640 metros sobre el nivel del mar (msnm) y procedentes de 12 distritos de Bogotá, de origen mestizo, español e indígena. La selección de la muestra fue de tipo no probabilística (cuotas). Los escolares tenían una condición socioeconómica media y los patrones alimentarios se basaban en el consumo cotidiano de productos, como la harina de maíz, papa, banana, col rizada y café.

Se excluyeron los alumnos que presentaban deficiencias físicas y los que no presentaron consentimiento (autorización) informado. Se incluyeron los escolares que previamente contaban con el consentimiento informado por los padres, asentimiento de los alumnos y los que completaron las mediciones. El estudio contó con el permiso de la Dirección del Colegio y se desarrolló de acuerdo con la Declaración de Helsinki y la Resolución 8430 del Ministerio de Salud y Protección Social de Colombia. Contó también con la aprobación del Comité de Ética local.

\section{Procedimientos}

El procedimiento de recolección de datos se organizó en tres etapas: en la primera, se explicaron los objetivos y las variables por evaluar a los padres, y se solicitó la firma del consentimiento informado. En la segunda, se organizaron las fichas de evaluación por edad, sexo, modalidad de estudio (primaria y/o secundaria), los horarios y responsables para la evaluación. En la tercera, se realizaron las evaluaciones en horario escolar de 7:00 a 12:00, de lunes a viernes durante el mes de octubre de 2013.

Las evaluaciones se efectuaron en las instalaciones del Colegio, a cargo de ocho profesores (cuatro hombres y cuatro mujeres), con amplia experiencia en mediciones antropométricas y que poseían capacitación previa por la Sociedad Internacional para el Avance de la Cineantropometría (International Society for the Advancement of Kinantoprometry, ISAK). ${ }^{13}$ El error técnica de medida (ETM) intra- e interobservador osciló entre 0,82\% y 1,5\%.

Las variables, como el peso y la estatura, fueron evaluadas con ropa ligera (descalzo, short y camiseta) según el protocolo estandarizado por la ISAK. ${ }^{13}$ Se evaluó el peso corporal $(\mathrm{kg})$ utilizando una balanza electrónica (Tanita, United Kingdom, Ltd), con una escala 0-150 kg y con precisión de $100 \mathrm{~g}$. La estatura, a través de un estadiómetro portátil (Seca Gmbh \& Co. KG, Hamburg, Germany), con una precisión de 0,1 $\mathrm{mm}$. El IMC fue calculado por la fórmula $\mathrm{IMC}=$ peso $(\mathrm{kg}) /$ estatura $^{2}(\mathrm{~m})$. Los intervalos de edades se organizaron de 6,0 a 6,9 años, de 7,0 a 7,9 años, hasta de 17,0 a 17,9 años.

Las comparaciones del peso, estatura e IMC fueron efectuadas con referencias internacionales: se utilizó la referencia de los CDC-2012, ${ }^{9}$ la referencia propuesta por Alfaro et al., ${ }^{1}$ para Jujuy (Argentina), para Campinas (Brasil) ${ }^{14} \mathrm{y}$ para la región central del Perú. ${ }^{15}$ Se optó por estas referencias por ser las más recientes y por presentar características regionales con similares índices de desarrollo humano (IDH) en los últimos años.

\section{Análisis estadístico}

La distribución normal de los datos fue verificada mediante la prueba de KolmogorovSmirnov. Posteriormente, se calculó la estadística descriptiva de media aritmética, desviación estándar (DE) y rango. Las diferencias entre ambos sexos se verificaron por medio del test " $t$ " para muestras independientes. Las 
diferencias entre la referencia de los CDC-2012 y los valores medios y \pm DE de Bogotá (Colombia) se determinaron por medio del test " $t$ " para muestras relacionadas. El nivel de significancia adoptado fue de 0,05 .

Las comparaciones con las referencias internacionales (Argentina, Brasil y Perú) se representaron gráficamente utilizando el percentil 50. Los percentiles (p5, p10, p15, p50, p85, p90 y p95) para el peso, estatura e IMC por rango de edad y sexo fueron desarrollados a partir del método LMS. ${ }^{16}$ Este permite la generación de curvas percentílicas específicas suavizadas: L (lambda; asimetría), M (mu; mediana) y S (sigma; coeficiente de variación). Se utilizó el software LMS Chart Maker versión 2.3. ${ }^{17}$ Los cálculos estadísticos fueron efectuados en planillas de Excel, SigmaStat y en SPSS 16.0.

\section{RESULTADOS}

Las variables que caracterizan a los 2241 escolares estudiados (1082 hombres y 1159 mujeres) se observan en la Tabla 1 . No hubo diferencias de peso corporal entre ambos sexos desde los 6 hasta los 14 años; sin embargo, desde los 15 hasta los 17 años, los hombres mostraron mayor peso corporal que sus contrapartes $(\mathrm{p}<0,05)$. En cuanto a la estatura, en edades iniciales (de 6 a 8 años), no hubo diferencias significativas, mientras que, en edades intermedias, las mujeres presentaron mayor estatura hasta los 12 años $(\mathrm{p}<0,05)$. Posteriormente, en edades avanzadas, los hombres evidenciaron mayor estatura en relación con las mujeres $(\mathrm{p}<0,05)$. Respecto al IMC, no hubo diferencias significativas entre ambos sexos desde los 6 hasta los 12 años. Por el contrario, desde los 13 hasta los 18 años, las mujeres presentaron valores significativamente más altos en relación con los hombres $(p<0,05)$.

Las comparaciones del peso y estatura de los escolares de Bogotá con la referencia de los CDC2012 y con otros estudios de América del Sur se observan en la Figura 1. En relación con el peso corporal, los escolares de ambos sexos de Bogotá presentaron similar patrón de crecimiento de los

TABLA 1. Características antropométricas de los escolares estudiados $(n=2241)$

\begin{tabular}{|c|c|c|c|c|}
\hline Edad (años) & $\begin{array}{c}\mathbf{n} \\
\text { Mujeres }\end{array}$ & $\begin{array}{c}\text { Peso }(\mathrm{kg}) \\
\mathrm{X} \pm \mathrm{DE}\end{array}$ & $\begin{array}{c}\text { Estatura (cm) } \\
X \pm D E\end{array}$ & $\begin{array}{c}\mathrm{IMC}\left(\mathrm{kg} / \mathrm{m}^{2}\right) \\
\mathrm{X} \pm \mathrm{DE}\end{array}$ \\
\hline $6,0-6,9$ & 102 & $21,1 \pm 3,9$ & $113,4 \pm 5,7$ & $16,3 \pm 2,0$ \\
\hline $7,0-7,9$ & 88 & $24,2 \pm 3,8$ & $121,6 \pm 4,7$ & $16,3 \pm 1,9$ \\
\hline $8,0-8,9$ & 82 & $27,9 \pm 5,4$ & $127,7 \pm 6,5$ & $17,1 \pm 2,5$ \\
\hline $9,0-9,9$ & 90 & $30,4 \pm 6,3$ & $133,4 \pm 6,4$ & $17,0 \pm 2,7$ \\
\hline $10,0-10,9$ & 70 & $34,1 \pm 6,2$ & $140,7 \pm 6,9$ & $18,1 \pm 2,5$ \\
\hline $11,0-11,9$ & 101 & $39,6 \pm 8,9$ & $146,5 \pm 6,6$ & $18,3 \pm 3,2$ \\
\hline $12,0-12,9$ & 79 & $41,3 \pm 9,5$ & $151,2 \pm 6,3$ & $19,3 \pm 3,3$ \\
\hline $13,0-13,9$ & 81 & $47,5 \pm 6,5$ & $155,7 \pm 4,9$ & $20,0 \pm 2,8$ \\
\hline $14,0-14,9$ & 96 & $53,2 \pm 8,7$ & $157 \pm 5,7$ & $21,5 \pm 3,1$ \\
\hline $15,0-15,9$ & 114 & $52,8 \pm 6,9$ & $158,2 \pm 5,8$ & $21,1 \pm 2,4$ \\
\hline $16,0-16,9$ & 105 & $52,9 \pm 7,9$ & $157,2 \pm 5,2$ & $21,4 \pm 2,4$ \\
\hline $17,0-17,9$ & 74 & $55,3 \pm 9,8$ & $158,1 \pm 5,5$ & $22,1 \pm 3,4$ \\
\hline \multirow[t]{2}{*}{ Total } & 1082 & $40 \pm 14,0$ & $143,5 \pm 16,6$ & $19,1 \pm 3,4$ \\
\hline & Hombres & & & \\
\hline $6,0-6,9$ & 120 & $21,3 \pm 31$ & $114,1 \pm 5,4$ & $16,4 \pm 1,7$ \\
\hline $7,0-7,9$ & 90 & $24,4 \pm 3,7$ & $120,9 \pm 5,4$ & $16,6 \pm 2,0$ \\
\hline $8,0-8,9$ & 93 & $27,9 \pm 5,5$ & $126,6 \pm 5,4$ & $17,3 \pm 2,8$ \\
\hline $9,0-9,9$ & 119 & $30,8 \pm 6,2$ & $131,4 \pm 5,7^{*}$ & $17,7 \pm 2,9$ \\
\hline $10,0-10,9$ & 86 & $34,1 \pm 7,3$ & $136,5 \pm 6,6^{*}$ & $18,2 \pm 2,8$ \\
\hline $11,0-11,9$ & 106 & $39,1 \pm 7,9$ & $143,8 \pm 7,6^{*}$ & $18,8 \pm 2,9$ \\
\hline $12,0-12,9$ & 100 & $42,9 \pm 7,7$ & $149,4 \pm 7,2^{*}$ & $19,2 \pm 2,5$ \\
\hline $13,0-13,9$ & 85 & $46,9 \pm 10,1$ & $157,5 \pm 8,4^{*}$ & $18,6 \pm 3,^{*}$ \\
\hline $14,0-14,9$ & 98 & $53,6 \pm 10,3$ & $164,5 \pm 6,9^{*}$ & $19,7 \pm 3,2^{*}$ \\
\hline $15,0-15,9$ & 90 & $56,21 \pm 9,6^{*}$ & $167,0 \pm 6,4^{*}$ & $20,1 \pm 3,0^{*}$ \\
\hline $16,0-16,9$ & 91 & $60,1 \pm 9,8^{*}$ & $168,1 \pm 5,9^{*}$ & $20,1 \pm 2,9^{*}$ \\
\hline $17,0-17,9$ & 81 & $61,3 \pm 9,3^{*}$ & $170,9 \pm 5,9^{*}$ & $20,6 \pm 2,9^{*}$ \\
\hline Total & 1159 & $40,8 \pm 15,7$ & $144,8 \pm 20,5$ & $18,7 \pm 3,1$ \\
\hline
\end{tabular}

IMC: índice de masa corporal; DE: desvío estándar. 
Figura 1. Comparación de los valores medios y \pm desvío estándar de las variables de peso y estatura de niños y adolescentes de Bogotá (Colombia) con la referencia americana de los Centros para el Control y la Prevención de Enfermedades 2012 y discrepancias entre el percentil 50 con estudios de América del Sur
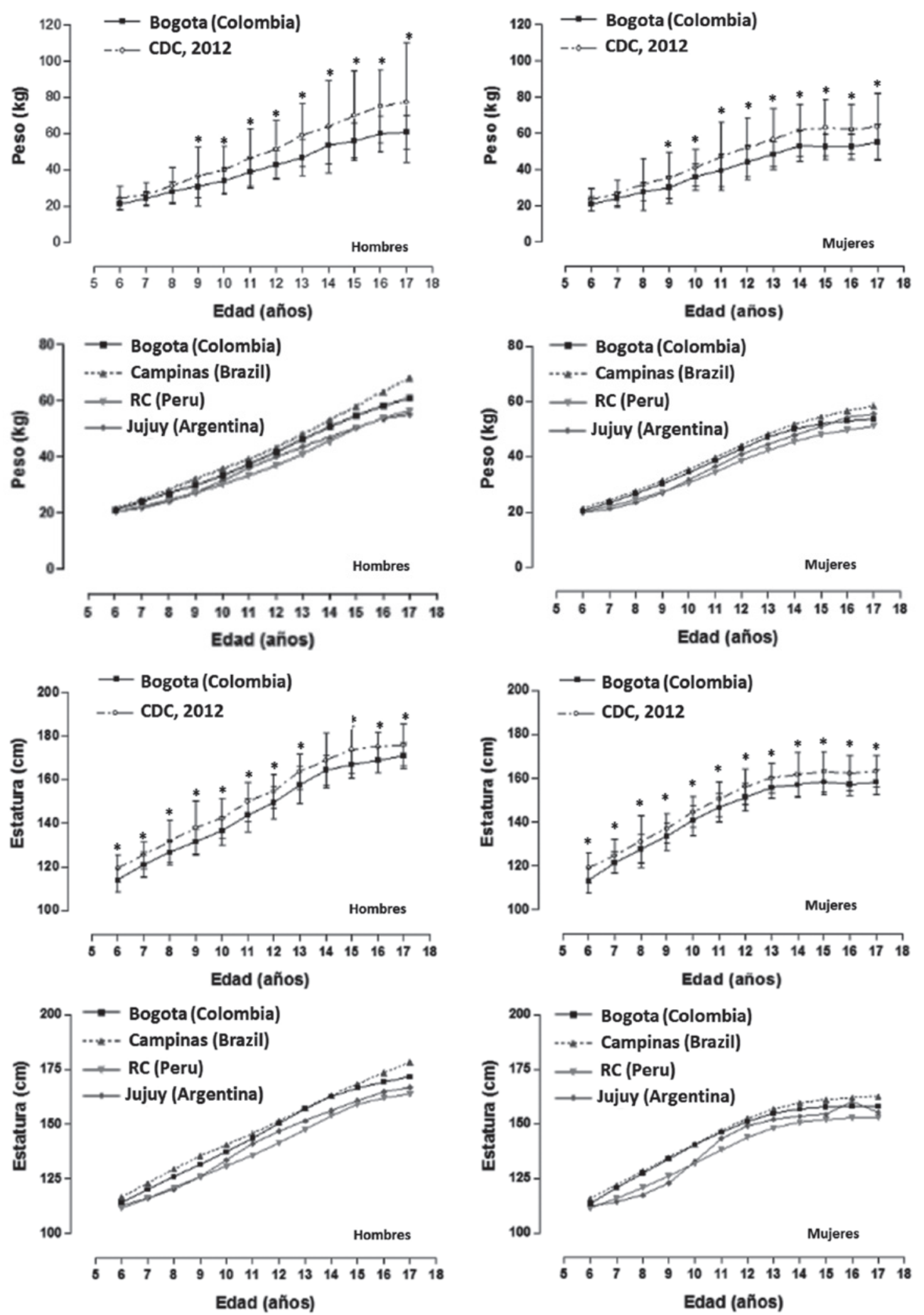

CDC: Centros para el Control y la Prevención de Enfermedades; RC: región central. 
6 hasta los 8 años. Luego, desde los 9 hasta los 17 años, los valores fueron significativamente inferiores a la referencia de los CDC-2012 $(\mathrm{p}<0,05)$. Se observaron también, en ambos sexos, valores de peso corporal relativamente similares con los estudios internacionales.

En la estatura, los escolares de ambos sexos de Bogotá presentaron valores inferiores en todas las edades en relación con la de los CDC-2012. En cuanto a las comparaciones con los estudios internacionales de América del Sur, se observaron patrones similares de crecimiento en ambos sexos, con ligeras discrepancias a lo largo de todas las edades.

Las comparaciones del IMC entre los escolares de Bogotá con referencias internacionales se observan en la Figura 2. En ambos sexos, los valores se incrementaron conforme la edad avanzaba. No hubo diferencias significativas con la referencia de los CDC-2012 en ambos sexos de los 6 hasta los 8 años; sin embargo, desde los 9 hasta los 17 años, las diferencias fueron significativas $(p<0,05)$ : los escolares de Bogotá mostraron valores inferiores de IMC. Respecto a las comparaciones con otros estudios internacionales, los hombres mostraron un similar comportamiento en todas las edades. Inclusive en las mujeres, este patrón fue similar en edades iniciales, pero, a partir de los 12 años, las discrepancias fueron mayores y se observaron valores inferiores de IMC.

La distribución de percentiles para el peso, estatura e IMC por edad y sexo se observa en la Tabla 2. En todos los casos, los valores mostraron un aumento creciente conforme la edad avanzaba. Los percentiles propuestos fueron p5, p10, p15, p50, p85, p90 y p95.

Las comparaciones gráficas de los percentiles (p5, p50 y p95) entre las curvas regionales

Figura 2. Comparación de los valores medios y \pm desvío estándar del índice de masa corporal de niños y adolescentes de Bogotá (Colombia) con la referencia americana de los Centros para el Control y la Prevención de Enfermedades 2012 y discrepancias entre el percentil 50 con estudios de América del Sur
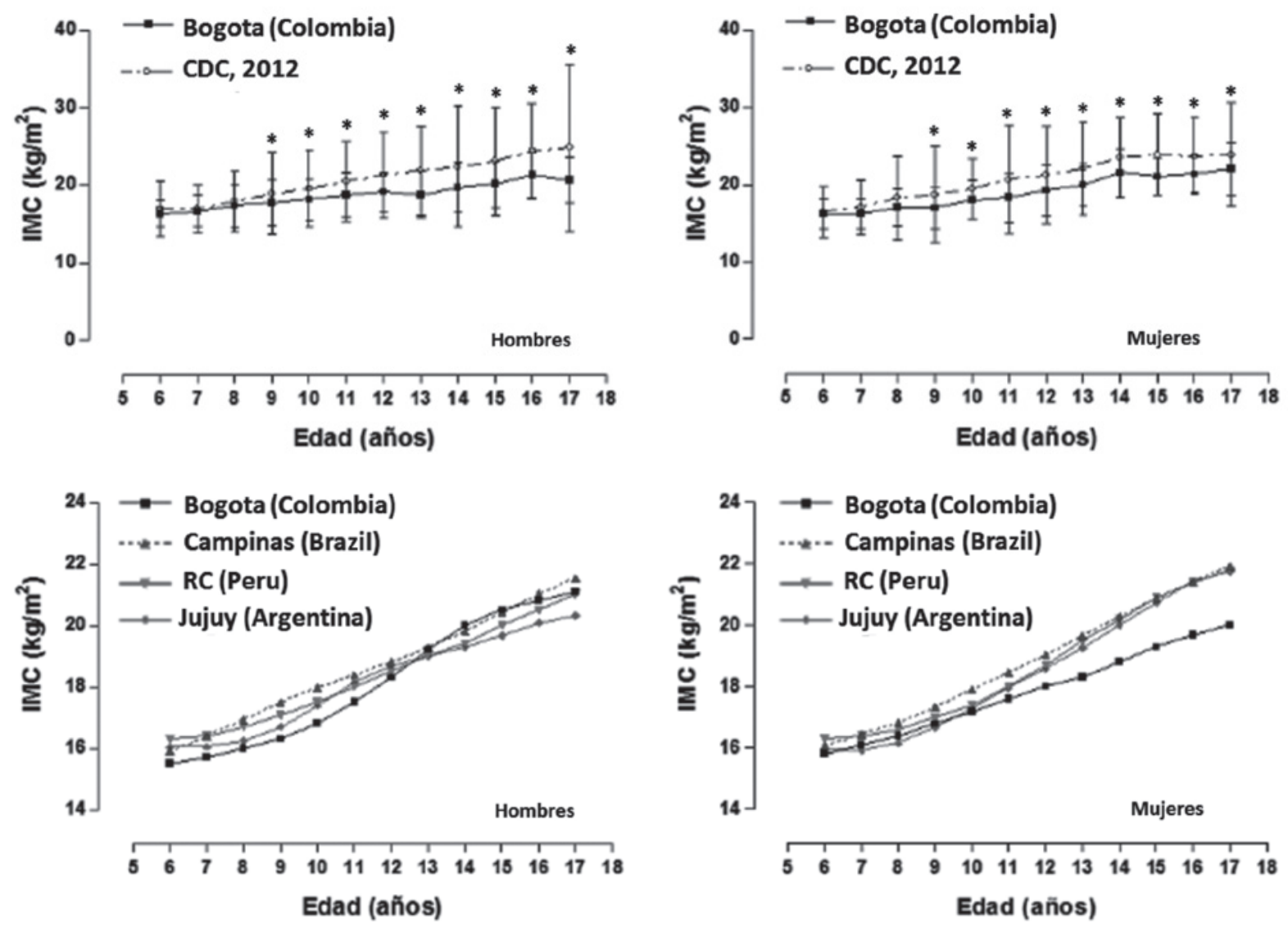

IMC: índice de masa corporal; CDC: Centros para el Control y la Prevención de Enfermedades; RC: región central. 


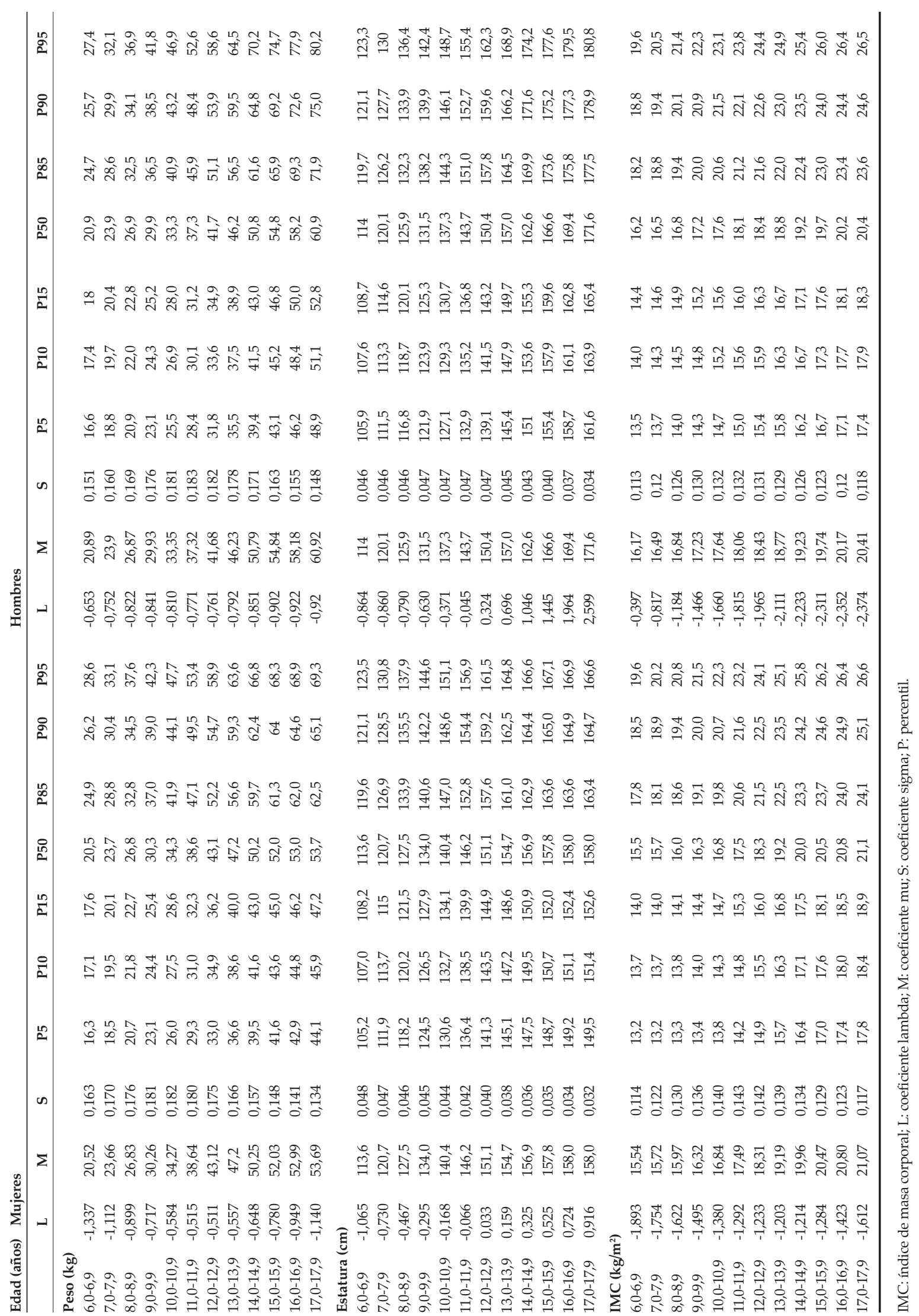


de Bogotá y de los CDC-2012 se observan en la Figura 3. Las curvas de peso, estatura e IMC mostraron patrones similares a las de los CDC-2012: los valores se incrementaron progresivamente conforme la edad cronológica aumentaba; sin embargo, fueron evidentes las discrepancias en todas las edades y en el p5, p50 y p95.

\section{DISCUSIÓN}

Este estudio es el primero en describir el CF de niños y adolescentes que viven a moderada altitud en Colombia. En general, los resultados analizados evidenciaron que los escolares de Bogotá de ambos sexos presentaron menor peso y estatura que la referencia de los CDC-2012.

De acuerdo con investigaciones previas, el
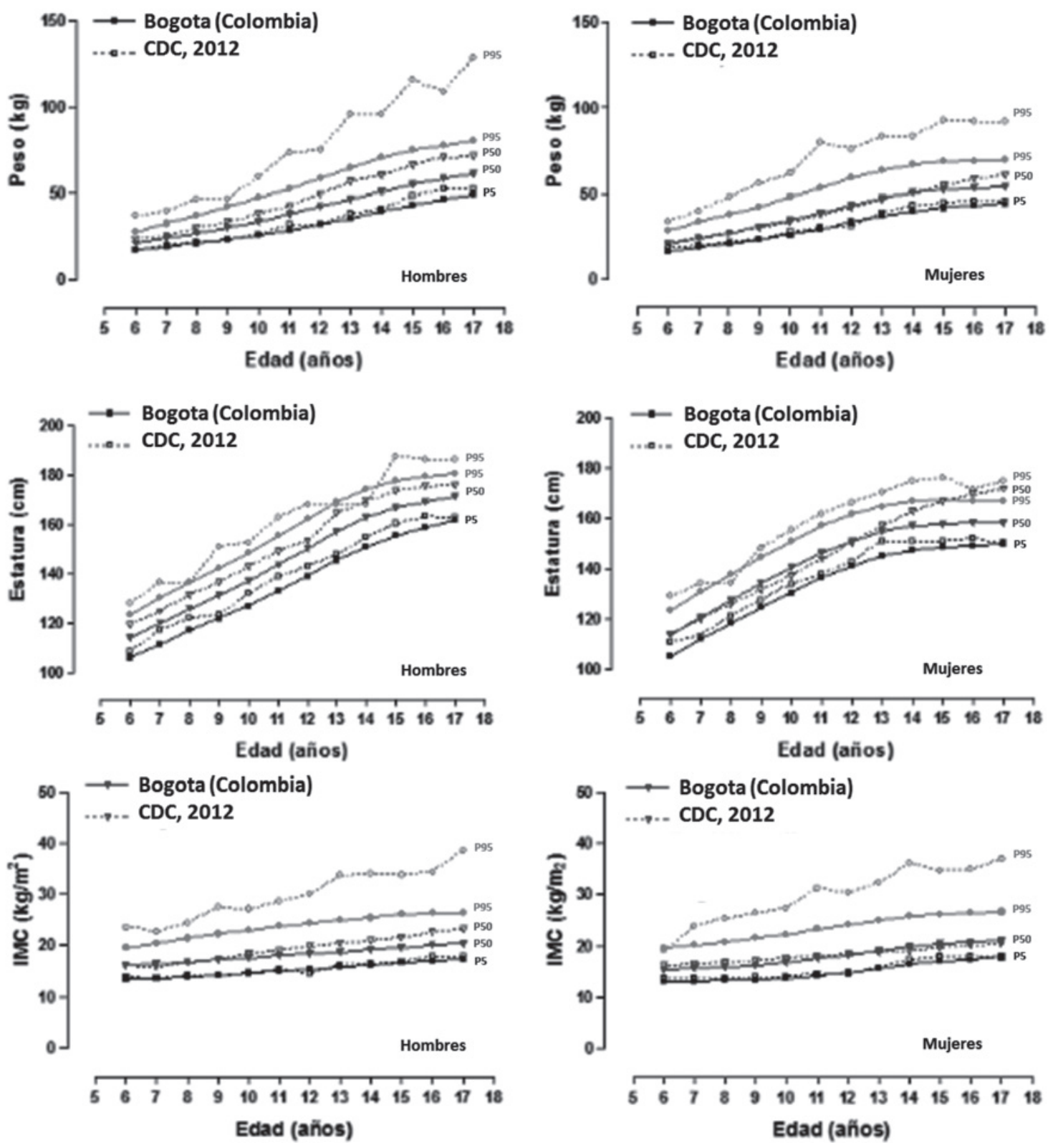

IMC: índice de masa corporal; CDC: Centros para el Control y la Prevención de Enfermedades; P: percentil. 
retraso en el CF en poblaciones de moderada ${ }^{18}$ y elevada altitud ${ }^{5}$ está fuertemente asociado a factores extrínsecos (medioambientales). Estos resultados confirman las diferencias culturales, medioambientales y genéticas específicas que las poblaciones de altitud suelen presentar. Además, cuando se comparó con otros estudios de América del Sur, ${ }^{1,4,15}$ los resultados evidenciaron patrones de CF relativamente inferiores.

Estos hallazgos confirman las discrepancias que existen en el peso y estatura entre escolares de diversas regiones geográficas del mundo, inclusive, es posible observar estas diferencias dentro de un mismo país en relación con la desigualdad de condiciones sociales, económicas, ambientales, así como los estilos de vida y el consumo de nutrientes entre regiones. ${ }^{19}$

Por lo tanto, los resultados observados subrayan la diversidad y dificultad de desarrollar estudios en ambientes complejos. ${ }^{20}$ Esto permite destacar la importancia de obtener nuevas descripciones de variables de CF y los valores de IMC entre poblaciones, sobre todo, en poblaciones escolares de moderada y elevada altitud.

De hecho, esta información sirve para comprender mejor la variación biológica humana y la salud pública ${ }^{3}$ de niños y adolescentes, puesto que, durante los primeros años de vida, resulta trascendental monitorizar el CF a través de la medición secuencial del peso, estatura e IMC. ${ }^{21}$ Esta práctica es habitual en América Latina, ${ }^{0}$ así como en Colombia y en otras partes del mundo.

Respecto a las comparaciones del IMC con la referencia de los CDC-2012, los resultados mostraron que los escolares que vivían a moderada altitud presentaban similares valores en edades iniciales $y$, posteriormente, desde los 9 hasta los 17 años, eran significativamente inferiores. Esto demuestra que el bajo peso y estatura conduce, ciertamente, a una disminución exagerada en los valores del IMC, producto de factores inherentes a grupos étnicos específicos que los países en desarrollo suelen experimentar (cambios económicos, nutricionales, culturales y ambientales).

De hecho, algunos estudios previos han demostrado que el IMC no es aplicable a niños y adolescentes de moderada altitud, ${ }^{18,23}$ puesto que, como índice de obesidad, no es posible su uso en poblaciones que tengan diferencias notables en estatura, ${ }^{24}$ en especial, en poblaciones que presentan retraso en el CF.

En ese sentido, la precisión del IMC para estimar el porcentaje de grasa corporal fue objeto de varios estudios. ${ }^{25,26} \mathrm{~A}$ pesar de ello, la $\mathrm{OMS}^{27}$ sugiere la utilización del IMC para la identificación y el diagnóstico del sobrepeso y obesidad de niños y adolescentes. Inclusive, en su diseño original, se incluyeron en la muestra los que nacieron por debajo de los $1500 \mathrm{msnm} .{ }^{28}$ Por lo tanto, las curvas de CF y los valores del IMC propuestos por la OMS no podrían ser aplicados en regiones de moderada altitud, como Bogotá (2640 msnm).

En consecuencia, ante las diferencias encontradas en peso, estatura e IMC con la referencia de los CDC-2012 y las discrepancias con estudios internacionales, se desarrollaron curvas percentílicas de peso, estatura e IMC por edad y sexo para evaluar el $\mathrm{CF}$, el estado nutricional y la adiposidad corporal de escolares de moderada altitud de Colombia.

En general, los percentiles de peso, estatura e IMC proporcionan información relevante para inferir el estado nutricional y de salud de niños y adolescentes ${ }^{29}$ independientemente de la región geográfica. Además, pueden ser utilizados para comparar con otras muestras internacionales y/o regionales con similares características, pues el valor real de las curvas reside en ayudar a determinar en qué grado las necesidades fisiológicas se están cumpliendo durante el proceso de crecimiento y desarrollo motor. ${ }^{30}$

Para tal efecto, se generaron percentiles, a través del método LMS, considerado una valiosa herramienta que presentaba claras ventajas en comparación con otros métodos de estimación tradicionales..$^{31}$ Los puntos de corte adoptados fueron los mismos utilizados en las referencias de los CDC 2000, 2012 y 2016..$^{8,9,32}$ Estos valores reflejaron categorías $(p<10$, de $\mathrm{p} 10$ a p85, de p85 a p95 y p > 95) de normal, sobrepeso y obesidad, respectivamente.

En esencia, Colombia presenta características orográficas y ambientales diversas, lo que lo constituye como un país atractivo para estudiar el CF. Inclusive en los últimos años, el IDH de Colombia ha ido incrementándose, ya que fue de 0,711 en el año 2013, y el de Bogotá fue de 0,911. ${ }^{33}$ Esto evidencia un rápido ritmo de crecimiento económico de Colombia, lo cual debe ser una preocupación creciente para monitorizar el CF.

Algunas fortalezas del estudio se destacan. Las evaluaciones fueron realizadas de forma estandarizada por evaluadores con amplia experiencia. Además, para construir las curvas de crecimiento, se consideró el ajuste de la altitud ${ }^{34}$ $\mathrm{y}$ algunos criterios que sugieren Butte et al., ${ }^{35}$ 
como la selección individual de la población, diseño de estudio, mediciones estandarizadas, modelos estadísticos. ${ }^{35}$ Sin embargo, el tamaño de la muestra fue determinado por medio de la selección de la muestra no probabilística, lo que, presumiblemente, podría limitar los resultados alcanzados. Se sugiere, para futuros estudios, efectuar un muestreo probabilístico, puesto que esto podría permitir la generalización a otros contextos regionales de Colombia.

En conclusión, las variables de peso, estatura e IMC difieren con la referencia de los CDC-2012 y con los estudios regionales de América del Sur. Estas discrepancias permitieron construir curvas de peso y estatura por edad y sexo para monitorizar el crecimiento de escolares de moderada altitud de Colombia. El IMC se utilizó en este estudio con fines informativos, ya que, como indicador de exceso de peso, es considerado inadecuado para escolares de moderada altitud. ${ }^{18}$ Los resultados sugieren su uso y aplicación en contextos clínicos y epidemiológicos.

http:/ / www.reidebihu.net/growth_bogota.php

\section{REFERENCIAS}

1. AlfaroE, Bejarano I, Dipierri J, et al. Percentilos de peso, talla e índice de masa corporal de escolares jujeños calculados por el método LMS. Arch Argent Pediatr 2004;102(6):431-9.

2. Guimarey LM, Carnese FR, Pucciarelli HM. La influencia ambiental en el crecimiento humano. Cienc Hoy 1995;5(30):41-7.

3. WHO Expert Committee. Physical Status: The Use and Interpretation of Anthropometry. Series 854, Geneva: WHO; 1995. [Acceso: 27 de septiembre de 2017]. Disponible en: http: / / apps.who.int/iris / bitstream/10665/37003/1/ WHO_TRS_854.pdf

4. Durán P, Merker A, Briceño G, et al. Colombian reference growth curves for height, weight, body mass index and head circumference. Acta Pædiatr 2016;105(3):e116-25.

5. PawsonI,Huicho L, Muro M, etal. Growth of childrenin two economically diverse Peruvian high-altitude communities. Am J Hum Biol 2001;13(3):323-40.

6. Cossio-Bolanos MA, Gomez-Campos R, Hespanhol JE, et al. Estudio del crecimiento físico de escolares a moderada altitud usando el área muscular del brazo por estatura y edad. Rev Andal Med Deporte 2013;6(2):66-72.

7. Cossio-Bolaños M, Gómez-Campos R, Andruske C, et al. Physical Growth, Biological Age, and Nutritional Transitions of Adolescents Living at Moderate Altitudes in Peru. Int $J$ Environ Res Public Health 2015;12(10):12082-94.

8. Kuczmarski R, Ogden C, Grummer-Strawn L, et al. CDC growth charts: United States. Advance Data 2000;314:1-28.

9. Fryar $\mathrm{CD}, \mathrm{Gu} \mathrm{Q}$, Ogden CL. Anthropometric reference data for children and adults: United States, 2007-2010. Vital Health Stat 2012;11(252):1-48.

10. World Health Organization. WHOChild GrowthStandards: Length/Height-for-Age, Weight-for-Age, Weight-forLength, Weight-for-Height and Body Mass Index-for-Age: Methods and Development. Geneva: WHO; 2006. [Acceso: 27 de septiembre de 2017]. Disponible en: http:/ / www. who.int/childgrowth/standards/Technical_report.pdf.

11. Lobstein T, Baur L, Uauy R. Obesity in children and young people: a crisis in public health. Obes Rev 2004;5(Suppl 1):4-104.

12. Rivera JA, González de Cossío T, Pedraza LS, et al. Childhood and adolescent overweight and obesity in Latin America: a systematic review. Lancet Diabetes Endocrinol 2014;2(4):321-32.

13. InternationalSociety for Advancementof Kinanthropometry (ISAK). International Standards for Anthropometric Assessment. Potchefstroom, RSA: ISAK, 2001. [Acceso: 27 de septiembre de 2017]. Disponible en: http: / / www.ceap. br/material/MAT17032011184632.pdf.

14. Gómez-Campos R, de Arruda M, Hespanhol JE, et al. Referencial values for the physical growth of school children and adolescents in Campinas, Brazil. Ann Hum Biol 2015;42(1):62-9.

15. Bustamante B, Freitas D, Pan H, et al. Centile Curves and Reference Values for Height, Body Mass, Body Mass Indexand Waist Circumference of Peruvian Children and Adolescents. Int J Environ Res Public Health 2015;12(3):2905-22.

16. ColeTJ, Bellizzi MC, Flegal KM, et al. Establishing a standard definition for child overweight and obesity worldwide: International survey. BMJ 2000;320(7244):1240-3.

17. Pan H, Cole TJ. LMSChartmaker. 2006. [Consulta: 28 de marzo de 2015]. Disponible en: http://www. healthforallchildren.co.uk.

18. Cossio-Bolaños MA, Viveros Flores A, Hespanhol JE, et al. Aplicabilidad del IMC en adolescentes escolares que viven a moderada altitud del Perú. Nutr Hosp 2015;31(2):922-7.

19. Cacciari E, Milani S, Balsamo A, et al. Italian cross-sectional growth charts for height, weight and BMI (2 to $20 \mathrm{yr}$ ). J Endocrinol Invest 2006;29(7):581-93.

20. Urlacher SS, Blackwell AD, Liebert MA, et al. Physical Growth of the Shuar: Height, Weight, and BMI References for an Indigenous Amazonian Population. Am J Hum Biol 2016;28(1):16-30.

21. Flores-Huerta S. Antropometría, estado nutricio y salud de los niños. Importancia de las mediciones comparadas. Bol Med Hosp Infant Mex 2005;63(2):73-5.

22. Comité nacional de crecimiento y desarrollo. La Sociedad Argentina de Pediatría actualiza las curvas de Crecimiento de niñas y niños menores de cinco años. Arch Argent Pediatr 2008;106(5):462-7.

23. Cossio-Bolaños MA, Santi Maria T, Gomez Campos R, et al. The use of World Health Organization growth curves in children and adolescents that live in regions of moderate altitude. Rev Paul Pediatr 2012;30(3):314-20.

24. Fernández López JA, Remesar X, Alemany M. Ventajas teóricas del índice de Rohrer (P/A3) sobre el índice de masa corporal (P/A2) para la estimación de la adiposidad en humanos. Rev Esp Obes 2005;3(1):47-55.

25. Pietrobelli A, Faith MS, Allison DB, et al. Body mass index as a measure of adiposity among children and adolescents: a validation study. J Pediatr 1998;132(2):204-10.

26. Mei Z, Grummer-Strawn LM, Pietrobelli A, et al. Validity of body mass index compared with other body-composition screening indexes for the assessment of body fatness in children and adolescents. Am J Clin Nutr 2002;75(6):978-85.

27. WHO Multicentre Growth Reference Study Group. Assessment of differences in linear growth among populations in the WHO Multicentre Growth Reference Study. Acta Paediatr Suppl 2006;450:56-65.

28. De Onis M, Onyango AW, Van den Broeck J, et al. Measurement and standardization protocols for anthropometry used in the construction of a new international growth reference. Food Nutr Bull 2004;25(Suppl 1):S27-36.

29. Grummer-Strawn LM, Garza C, Johnson CL. Childhood growth charts. Pediatrics 2002;109(1):141-2. 
e250 / Arch Argent Pediatr 2018;116(2):e241-e250 / Artículo original

30. De Onis M. Growth curves for school age children and adolescents. Indian Pediatr 2009;46(6):463-5.

31. Roelants M,Hauspie R, Hoppenbrouwers K. References for growth and pubertal development from birth to 21 years in Flanders, Belgium. Ann Hum Biol 2009:36(6):680-94.

32. Fryar $\mathrm{CD}, \mathrm{GuQ}$, Ogden CL, et al. Anthropometric reference data for children and adults: United States, 2011-2014. Vital Health Stat 2016;3(39):1-46.

33. Informe Sobre Desarrollo Humano 2013. El ascenso del Sur: Progreso humano en un mundo diverso. New York UNDP; 2013. [Acceso:27 de septiembre de2017]. Disponible en:http:/ / www.undp.org/content/dam/undp/library/ corporate/HDR/2013GlobalHDR/Spanish/HDR2013\%20 Report\%20Spanish.pdf.

34. Ponce de León S. The WHO Multicentre growth reference study and altitude above sea level. An example of hypsometric bias? High Alt Med Biol 2008;9(3):249-51.

35. Butte NF, Garza C, De Onis M. Evaluation of the feasibility of international growth standards for school-aged children and adolescents. Food Nutr Bul. 2006;27(4 Suppl Growth Standard):S169-74. 